\title{
Remaining useful life prediction methods for key structures of heavy-duty railway wagons based on condition monitoring data
}

\author{
Chao Wang ${ }^{1}$, Tao Zhu ${ }^{1}$, Bing Yang ${ }^{1}$, Minxuan Yin ${ }^{1}$, Shoune Xiao ${ }^{1}$, and GUANGWU \\ YANG $^{1}$
}

${ }^{1}$ State Key Laboratory of Traction Power

December 25, 2021

\begin{abstract}
To predict the remaining useful life for the key structures of heavy-duty railway wagons using condition monitoring data, methods for the coupler body with and without visible cracks were proposed. First, a method based on the delay time and hypothesis testing was proposed, considering the case without visible cracks in the coupler body. Then, for the case of visible cracks, methods based on a hypothetical distribution and support vector regression with the Kalman filter were proposed. Finally, by taking the coupler body monitoring data as an example, the prediction accuracies of the proposed methods were compared. The results indicated that the prediction method that only considers the common characteristics of the research objects had an average relative error of $57.56 \%$ for the coupler structure with a long lifespan. Considering the delay time of the current state of the structure and the assumed distribution prediction method, the relative error was reduced to $34.52 \%$, and the remaining useful life prediction value fluctuated sharply with respect to the service mileage. On this basis, considering the performance degradation process of the structure, the change in the remaining useful life prediction value was smoother, and the relative error was $43.67 \%$. The methods for predicting the remaining useful life of railway heavy-duty coupler bodies using condition monitoring data have important theoretical and practical value for improving vehicle safety, reducing maintenance costs, and accurately evaluating the remaining useful life.
\end{abstract}

\section{Hosted file}

Manuscript.docx available at https://authorea.com/users/452631/articles/550630-remaininguseful-life-prediction-methods-for-key-structures-of-heavy-duty-railway-wagons-based-oncondition-monitoring-data 